\title{
Interaction of high-velocity pulsars with supernova remnant shells
}

\author{
E. van der Swaluw ${ }^{1,2}$, A. Achterberg ${ }^{1}$, Y. A. Gallant ${ }^{1,3}$, T. P. Downes ${ }^{4}$, and R. Keppens ${ }^{5}$ \\ 1 Astronomical Institute, Utrecht University, PO Box 80000, 3508 TA Utrecht, The Netherlands \\ 2 Dublin Institute for Advanced Studies, 5 Merrion Square, Dublin 2, Ireland \\ 3 Service d'Astrophysique, CEA Saclay, 91191 Gif-sur-Yvette Cedex, France \\ ${ }^{4}$ School of Mathematical Sciences, Dublin City University, Glasnevin, Dublin 9, Ireland \\ 5 FOM-Institute for Plasma Physics Rijnhuizen, PO Box 1207, 3430 BE Nieuwegein, The Netherlands
}

Received 4 February 2002 / Accepted 7 October 2002

\begin{abstract}
Hydrodynamical simulations are presented of a pulsar wind emitted by a supersonically moving pulsar. The pulsar moves through the interstellar medium or, in the more interesting case, through the supernova remnant created at its birth event. In both cases there exists a three-fold structure consisting of the wind termination shock, contact discontinuity and a bow shock bounding the pulsar wind nebula. Using hydrodynamical simulations we study the behaviour of the pulsar wind nebula inside a supernova remnant, and in particular the interaction with the outer shell of swept up interstellar matter and the blast wave surrounding the remnant. This interaction occurs when the pulsar breaks out of the supernova remnant. We assume the remnant is in the Sedov stage of its evolution. Just before break-through, the Mach number associated with the pulsar motion equals $\mathcal{M}_{\mathrm{psr}}=7 / \sqrt{5}$, independent of the supernova explosion energy and pulsar velocity. The bow shock structure is shown to survive this break-through event.
\end{abstract}

Key words. pulsars: general - supernova remnants - shock waves - hydrodynamics

\section{Introduction}

A supernova explosion of a massive star will result in an expanding supernova remnant (SNR). In some cases the fossil of the progenitor star is a pulsar moving at high velocity. Even though the precise physical mechanism responsible for imparting a large kick velocity to single radio pulsars at birth has not been identified, observations of the pulsar distribution with respect to the mid-plane of the galaxy indicate that they are born with a velocity in the range $V_{\mathrm{psr}} \sim 100-1000 \mathrm{~km} \mathrm{~s}^{-1}$ (Harrison et al. 1993; Lyne \& Lorimer 1994). A similar range of values is obtained from a sample of SNR-pulsar associations (Frail et al. 1994).

The expansion of a SNR is decelerated due to mass-loading by swept up interstellar medium (ISM) or by material from a progenitor wind. As the pulsar moves with a constant velocity it will ultimately break through the SNR shell. Two observed systems are often presented as an illustration of this scenario:

CTB80: in this supernova remnant the pulsar PSR B1951+32 is located (in projection) just inside the outer edge of the remnant. The spectral index of the synchrotron emission in the vicinity of the pulsar system indicates that there is a plerionic nebula around the pulsar, see for example Strom (1987) and Migliazzo et al. (2002).

Send offprint requests to: E. van der Swaluw, e-mail: swaluw@cp.dias.ie
G5.4-1.2: in this case the pulsar is located well outside the supernova remnant. At radio frequencies an emission bridge appears to connect the pulsar B1757-24 and the associated pulsar wind nebula $(\mathrm{PWN})$ with the supernova remnant (Frail \& Kulkarni 1991), suggesting a physical association between the supernova remnant and the pulsar. It should be pointed out, however, that a new upper limit on the proper motion of B175724 (Gaensler \& Frail 2000) puts the component of the pulsar velocity in the plane of the sky at $V_{\perp \mathrm{psr}} \leq 600 \mathrm{~km} \mathrm{~s}^{-1}$ for an assumed distance of $5 \mathrm{kpc}$. This leads to a discrepancy between the characteristic pulsar age, obtained from its spin period derivative $(P / 2 \dot{P} \sim 16 \mathrm{kyr})$, and the dynamical age obtained from the offset distance $R_{\mathrm{psr}}$ from the center of G5.4-1.2 $\left(R_{\mathrm{psr}} / V_{\mathrm{psr}} \geq 39 \mathrm{kyr}\right)$.

Both systems are clearly brightened at radio wavelengths near the position of the pulsar, and it has been suggested that the associated pulsar wind is rejuvenating the radio emission from the SNR shell by the injection of fresh relativistic electrons (Shull et al. 1989). In this paper we will investigate the hydrodynamical aspects of the interaction between a pulsar wind and a SNR shell.

Most pulsars have a radio lifetime $\left(10^{6}-10^{7} \mathrm{yr}\right)$ which is much larger than the age $<10^{4}$ yr of a SNR in the Sedov phase. Therefore pulsars will remain visible long after the associated SNR has dissolved into the interstellar medium. The pulsar then moves as an isolated pulsar through the interstellar 
medium, and can form a pulsar wind nebula bow shock system. A typical example of such a system is the Guitar Nebula around PSR B2224+65 which has been detected both in $\mathrm{H} \alpha$ (Cordes et al. 1993) and in X-rays (Romani et al. 1997), but which has no associated SNR.

In this paper we consider the case where the pulsar's kick velocity is sufficiently high so that it leaves the supernova remnant while it is still in the Sedov stage. We describe three different stages in the evolution of the pulsar-SNR system: (1) the stage where the PWN/bow shock resides inside the SNR, (2) the PWN/bow shock breaking through the shell of the SNR and (3) the stage where the PWN/bow shock moves through the ISM.

\section{Physics of a PWN bow shock inside a SNR}

\subsection{Dynamics of the pulsar/SNR system}

In rapidly rotating (young or recycled) pulsars, it is believed that a pulsar wind carries away most of the spindown luminosity,

\section{$L=I \Omega \dot{\Omega}$}

of a pulsar with rotation period $P=2 \pi / \Omega$ and moment of inertia $I$. This relativistic wind is presumably generated in the pulsar magnetosphere, and accelerates electrons, positrons and possibly nuclei to ultra-relativistic speeds.

The pulsar wind blows a bubble or pulsar wind nebula (PWN) into the surrounding medium. This PWN is initially located well within the interior of the SNR created at the birth of the neutron star. During the free expansion stage of the SNR evolution the typical expansion speed of the stellar ejecta as determined by the mechanical energy $E_{\mathrm{snr}}$ released in the explosion and the ejecta mass $M_{\mathrm{ej}}$,

$V_{\mathrm{ej}} \sim \sqrt{\frac{10}{3} \frac{E_{\mathrm{snr}}}{M_{\mathrm{ej}}}} \sim 10000 \mathrm{~km} \mathrm{~s}^{-1}$,

is generally much larger than the kick velocity of the pulsar. As a result the PWN is located relatively close to the center of the SNR at this stage. In this regime, the results of our earlier, spherically symmetric simulations (van der Swaluw et al. 2001) are expected to remain approximately valid. Only when the SNR expansion slows down as it enters the Sedov stage after some $\sim 500-1000 \mathrm{yr}$, a situation is possible where the pulsar position becomes strongly off-center with respect to the SNR.

The Sedov stage of SNR expansion lasts until internal (radiative) cooling becomes important. The SNR then enters the so-called pressure-driven snowplow (PDS) stage. The relevant transition time is calculated by Blondin et al. (1998):

$t_{\mathrm{PDS}}=2.9 \times 10^{4} E_{51}^{4 / 17} n_{0}^{-9 / 17} \mathrm{yr}$.

Here $E_{51}$ is the explosion energy $E_{\mathrm{snr}}$ in units of $10^{51} \mathrm{ergs}$ and $n_{0}$ denotes the hydrogen number density in the ISM.

We will describe the physics of a pulsar wind interaction with the shell of a SNR in the Sedov stage. Consequently the results presented below only apply for pulsar velocities $V_{\mathrm{psr}}$ larger than a minimum value. Equating the distance traveled by the pulsar,

$R_{\mathrm{psr}}=V_{\mathrm{psr}} t$

with the radius for a SNR embedded in a homogeneous interstellar medium of density $\rho_{\text {ism }}$ in the Sedov stage,

$R_{\mathrm{snr}} \simeq 1.15\left(\frac{E_{\mathrm{snr}}}{\rho_{\mathrm{ism}}}\right)^{1 / 5} t^{2 / 5}$,

one gets the crossing time for the pulsar:

$t_{\mathrm{cr}}=1.27\left(\frac{E_{\mathrm{snr}}}{\rho_{\mathrm{ism}} V_{\mathrm{psr}}^{5}}\right)^{1 / 3}$,

which is

$t_{\text {cr }} \simeq 1.4 \times 10^{4} E_{51}^{1 / 3} V_{1000}^{-5 / 3} n_{0}^{-1 / 3} \mathrm{yr}$.

Here $V_{1000}$ is the velocity of the pulsar in units of $1000 \mathrm{~km} \mathrm{~s}^{-1}$, and $n_{0}$ the hydrogen number density of the ISM in units of $\mathrm{cm}^{-3}$. The requirement $t_{\mathrm{cr}} \leq t_{\mathrm{PDS}}$ yields the minimum velocity a pulsar needs in order to break out of the SNR while the latter is still in the Sedov stage:

$V_{\mathrm{psr}} \geq 650 n_{0}^{2 / 17} E_{51}^{1 / 17} \mathrm{~km} \mathrm{~s}^{-1}$.

An analysis of observed pulsar velocities by Hansen \& Phinney (1997) find a distribution which is consistent with a Maxwellian with dispersion in the velocities ("temperature") equal to $\sigma_{\mathrm{v}}=190 \mathrm{~km} \mathrm{~s}^{-1}$. If this is the case, only about $1 \%$ of all pulsars acquires a kick velocity large enough to satisfy condition (5). It should be noted however that the distribution at the high-velocity end is not tightly constrained by observations, and the fraction could be much higher. A more recent analysis of available data by Arzoumanian et al. (2002) finds strong evidence of a double-peaked distribution of pulsar birth velocities, with characteristic velocities of $\sim 90 \mathrm{~km} \mathrm{~s}^{-1}$ and $\sim 500 \mathrm{~km} \mathrm{~s}^{-1}$ respectively, and a much higher fraction of high-velocity pulsars. They find that up to $10 \%$ of the pulsars younger than $20 \mathrm{kyr}$ will be found outside the supernova remnant left by the progenitor.

One can use the Rankine-Hugoniot relations to determine the pressure just behind the Sedov-Taylor blast wave bounding the SNR (assuming a strong shock and a gas with specific heat ratio $\gamma=5 / 3$ )

$P_{\text {sh }}=\frac{3}{4} \rho_{\text {ism }} V_{\text {snr }}^{2}$,

where

$V_{\mathrm{snr}} \equiv \frac{\mathrm{d} R_{\mathrm{snr}}}{\mathrm{d} t}=\frac{2}{5} \frac{R_{\mathrm{snr}}}{t}$

is the SNR expansion speed. Using this expression along with $R_{\mathrm{snr}}=R_{\mathrm{psr}}$ and the strong shock jump conditions, one can derive the following equations valid at the moment of breakthrough. The speed of the pulsar is related to the SNR expansion speed by

$V_{\mathrm{psr}}=\frac{5}{2} V_{\mathrm{snr}}$, 
while the material in the shell behind the SNR blast wave moves with a velocity

$V_{\mathrm{sh}}=\frac{3}{4} V_{\mathrm{snr}}$.

This corresponds to a relative speed between pulsar and postshock material equal to

$V_{\text {rel }} \equiv V_{\mathrm{psr}}-V_{\mathrm{sh}}=\frac{7}{4} V_{\mathrm{snr}}$.

The density in the shell is roughly the post-shock density for a strong shock, $\rho_{\mathrm{sh}}=4 \rho_{\text {ism }}$, so the Mach number $\mathcal{M}_{\mathrm{psr}}$ of the pulsar motion through the shell material satisfies

$\mathcal{M}_{\mathrm{psr}}=\frac{V_{\mathrm{rel}}}{\sqrt{\gamma P_{\mathrm{sh}} / 4 \rho_{\mathrm{ism}}}}=\frac{7}{\sqrt{5}} \approx 3.13$.

Since the proper motion of the pulsar is supersonic with respect to the surrounding medium the outer rim of the PWN will become a-spherical and a bow shock will form.

After the end of the Sedov phase, when the swept-up material in the shell behind the blast wave cools radiatively, the outward motion of the shell is driven by the pressure of the adiabatically cooling interior, with $P \propto \rho^{\gamma} \propto R^{-3 \gamma}$. The balance between the ram pressure and the interior pressure, $P \sim \rho_{\text {ism }} V_{\mathrm{s}}^{2}$, leads to an expansion law $R_{\mathrm{S}} \propto t^{\eta}$, with $\eta=2 /(3 \gamma+2)=2 / 7$ for $\gamma=5 / 3$. Calculations of Cioffi et al. (1988) show that the remnant interior is slightly over-pressured, and consequently the expansion proceeds with $\eta \approx 3 / 10$ for $t \gg t_{\mathrm{PDS}}$. Cioffi et al. (1988) fit the results of their simulations for $t_{\mathrm{PDS}}<t<35 t_{\mathrm{PDS}}$ with an expansion law

$R_{\mathrm{snr}}(t)=R_{\mathrm{PDS}}\left(\frac{4}{3} \frac{t}{t_{\mathrm{PDS}}}-\frac{1}{3}\right)^{3 / 10}$.

We adopt this expansion law, but using the transition time $t_{\mathrm{PDS}}$ from Blondin et al. (1998) which is $20 \%$ larger than the one used originally by Cioffi and coworkers. with

$R_{\mathrm{PDS}}=1.15\left(\frac{E_{\mathrm{snr}}}{\rho_{\mathrm{ism}}}\right)^{1 / 5} t_{\mathrm{PDS}}^{2 / 5}=18 E_{51}^{5 / 17} n_{0}^{-7 / 17} \mathrm{pc}$.

The crossing time $t_{\mathrm{cr}}$ is determined by the dimensionless parameter

$\alpha_{\mathrm{PDS}} \equiv \frac{R_{\mathrm{PDS}}}{V_{\mathrm{psr}} t_{\mathrm{PDS}}}=0.64 E_{51}^{1 / 17} n_{0}^{2 / 17} V_{1000}^{-1}$,

where the pulsar crosses the shell in the snowplow phase if $\alpha_{\text {PDS }}>1$. Note that this quantity depends only weakly on the explosion energy, and on the metallicity and density of the surrounding interstellar medium. Figure 1 gives a graphical representation of the expansion of the remnant and the pulsar motion. It shows that the crossing occurs at $t_{\mathrm{cr}} \sim 2 t_{\mathrm{PDS}}$ for $\alpha_{\mathrm{PDS}} \approx 1.5$, at $t_{\mathrm{cr}} \sim 3 t_{\mathrm{PDS}}$ for $\alpha_{\mathrm{PDS}} \approx 2$ and at $t_{\mathrm{cr}} \sim 4 t_{\mathrm{PDS}}$ for $\alpha_{\mathrm{PDS}} \approx 2.5$. For typical parameters this corresponds to a pulsar velocity equal to $V_{\mathrm{psr}} \sim 430 \mathrm{~km} \mathrm{~s}^{-1}, V_{\mathrm{psr}} \sim 315 \mathrm{~km} \mathrm{~s}^{-1}$ and $V_{\mathrm{psr}} \sim 260 \mathrm{~km} \mathrm{~s}^{-1}$ respectively.

The ratio of the pulsar velocity and the shock speed, $V_{\mathrm{psr}} / V_{\mathrm{snr}}$ increases slightly from $V_{\mathrm{snr}} / V_{\mathrm{psr}}=2.5$ if $\alpha_{\mathrm{PDS}}=1$ to $V_{\mathrm{psr}} / V_{\mathrm{snr}} \sim 3$ for $\alpha_{\mathrm{PDS}} \gg 1$. However, in the radiative shell, the density is much larger than the density immediately behind the pressure-driven snowplow

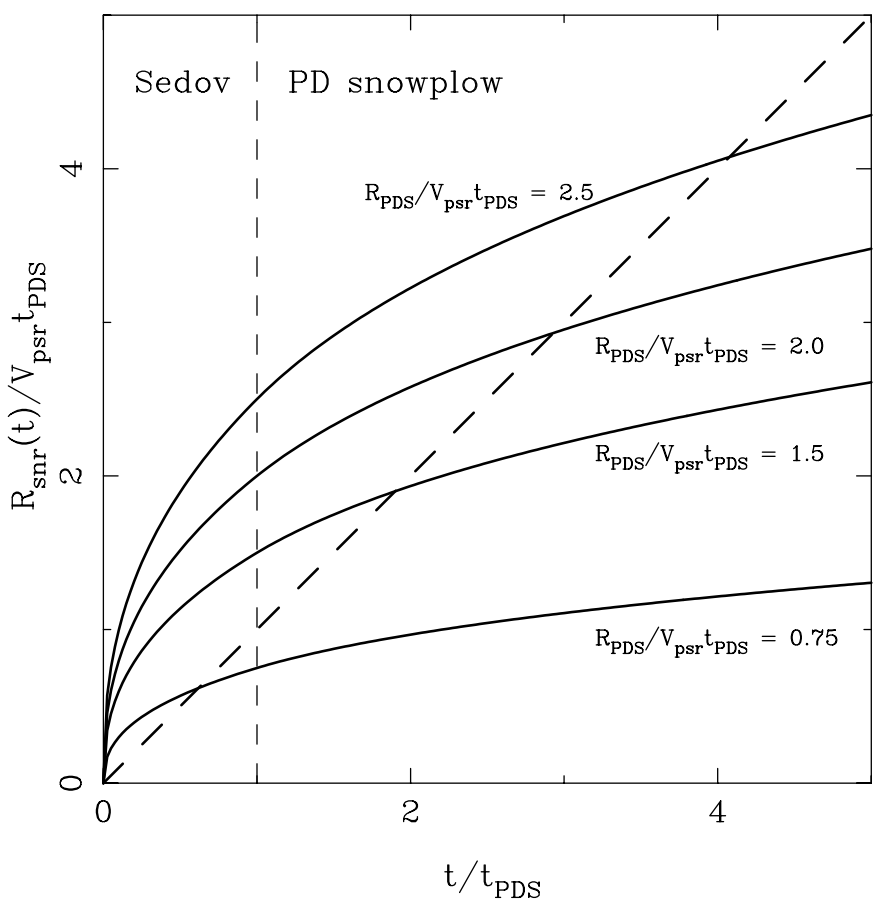

Fig. 1. The expansion of a supernova remnant in the pressure-driven snowplow phase. The solid curves show the radius of the supernova remnant in units of the distance travelled by the pulsar in a time $t_{\mathrm{PDS}}$, the transition time to the pressure-driven snowplow phase. Curves are shown for different values of the parameter $\alpha_{\mathrm{PDS}}=R_{\mathrm{PDS}} / V_{\mathrm{psr}} t_{\mathrm{PDS}}$. The dashed diagonal line gives the position of the pulsar. In the case $\alpha_{\text {PDS }}<$ 1 the pulsar crosses the remnant while it is still in the Sedov phase.

blast wave, and the pressure and temperature is much smaller due to cooling. The associated reduction of the sound speed in the shell implies that the motion of the pulsar through the shell will be highly supersonic.

\subsection{Pulsar wind}

A pulsar wind is believed to consist of an ultra-relativistic, cold flow with a large bulk Lorentz factor, e.g. $\Gamma_{\mathrm{w}} \geq 10^{3}$ in the case of the Crab (Gallant et al. 2002). The cold wind is terminated by a termination shock which thermalizes the flow, leading to a relativistically hot downstream state with sound speed $s \sim$ $c / \sqrt{3}$. Following Kennel \& Coroniti (1984), the luminosity of the pulsar wind at the wind termination shock $R_{\mathrm{ts}}$, is given by a combination of particle and magnetic luminosity:

$L=4 \pi \Gamma_{\mathrm{w}}^{2} n_{\mathrm{w}} R_{\mathrm{ts}}^{2} m c^{3}(1+\sigma)$,

where $n_{\mathrm{w}}$ is the proper density in the wind, $m$ the mean mass per particle and $\sigma$ is the ratio of Poynting flux to plasma kinetic energy flux. The parameter $\sigma$ for pulsar winds is thought to be small, therefore we consider an unmagnetised pulsar wind, i.e. $\sigma=0$. The typical pressure behind the ultra-relativistic termination shock is then (e.g. Kennel \& Coroniti 1984)

$P_{\mathrm{ts}} \approx \frac{2}{3} \Gamma_{\mathrm{w}}^{2} n_{\mathrm{w}} m c^{2}=\frac{L}{6 \pi R_{\mathrm{ts}}^{2} c}$. 
The first equality is approximate because of deviations of sphericity of the pulsar wind region, induced by the proper motion of the pulsar. The shocked pulsar wind material is separated from material that has gone through the bow shock by a contact discontinuity. Because of the high internal sound speed, both in the pulsar wind material and in the shocked material behind the bow shock, perturbations in the pressure between the bow shock, the stagnation point on the contact discontinuity, and the pulsar wind termination shock are smoothed out rapidly. This high-pressure region is relatively thin, and can be considered as isobaric to lowest approximation. Our simulations bear this out (e.g. Fig. 7). We obtain the pressure behind the head of the bow shock at break-through, using the RankineHugoniot jump conditions with adiabatic index $\gamma=5 / 3$ for a shock with a Mach number $\mathcal{M}_{\mathrm{psr}}$ :

$P_{\mathrm{bs}}=\left(5 \mathcal{M}_{\mathrm{psr}}^{2}-1\right) P_{\mathrm{sh}} / 4=9 \rho_{\mathrm{ism}} V_{\mathrm{snr}}^{2}$.

After the pulsar has broken through the shell the pulsar wind is completely confined by the ram pressure of the cold ISM and the stagnation-point pressure, using the Rankine-Hugoniot relations for a strong shock, drops to

$P_{\mathrm{bs}}=\frac{3}{4} \rho_{\mathrm{ism}} V_{\mathrm{psr}}^{2}=\frac{75}{16} \rho_{\mathrm{ism}} V_{\mathrm{snr}}^{2}$,

a pressure reduction by roughly $50 \%$ as the pulsar leaves the SNR. The fact that the region between termination shock and bow shock is almost isobaric implies

$P_{\mathrm{bs}} \approx P_{\mathrm{ts}}$.

This condition, together with Eq. (15), determines the standoff distance of the pulsar wind termination shock as

$R_{\mathrm{ts}}=\eta\left(\frac{L}{6 \pi \rho_{\mathrm{ism}} V_{\mathrm{psr}}^{2} c}\right)^{1 / 2}$,

where the numerical factor $\eta$ is determined by Eqs. (16) or (17). This parameter takes the value $\eta=5 / 6 \approx 0.83$ when the pulsar is still just inside the SNR, and $\eta=2 / \sqrt{3} \approx 1.15$ when the pulsar moves through the ISM. The radius $R_{\mathrm{ts}}$ is also the typical standoff distance of the bow shock, which is always close to the termination shock at the head of the pulsar wind nebula.

These expressions allow us to calculate the relative size of the pulsar wind to the supernova remnant at the moment of break-through. From the expression (3) for the crossing time one has

$R_{\mathrm{snr}}\left(t_{\mathrm{cr}}\right)=V_{\mathrm{psr}} t_{\mathrm{cr}}=13.6 E_{51}^{1 / 3} V_{1000}^{-2 / 3} n_{0}^{-1 / 3} \mathrm{pc}$.

The termination shock radius is of order

$R_{\mathrm{ts}} \simeq 2.8 \times 10^{-3} \eta L_{36}^{1 / 2} n_{0}^{-1 / 2} V_{1000}^{-1} \mathrm{pc}$,

here $L_{36}=L /\left(10^{36} \mathrm{erg} / \mathrm{s}\right)$. Note that the size of the SNR shell is much larger then the size of the PWN. For this reason we will neglect the curvature of the SNR blast wave and perform a hydrodynamical simulation where the pulsar moves with a Mach number of $\mathcal{M}_{\mathrm{psr}}=7 / \sqrt{5} \simeq 3.13$ through the post-shock flow of a strong plane-parallel shock, ultimately crossing this shock into the unshocked medium.
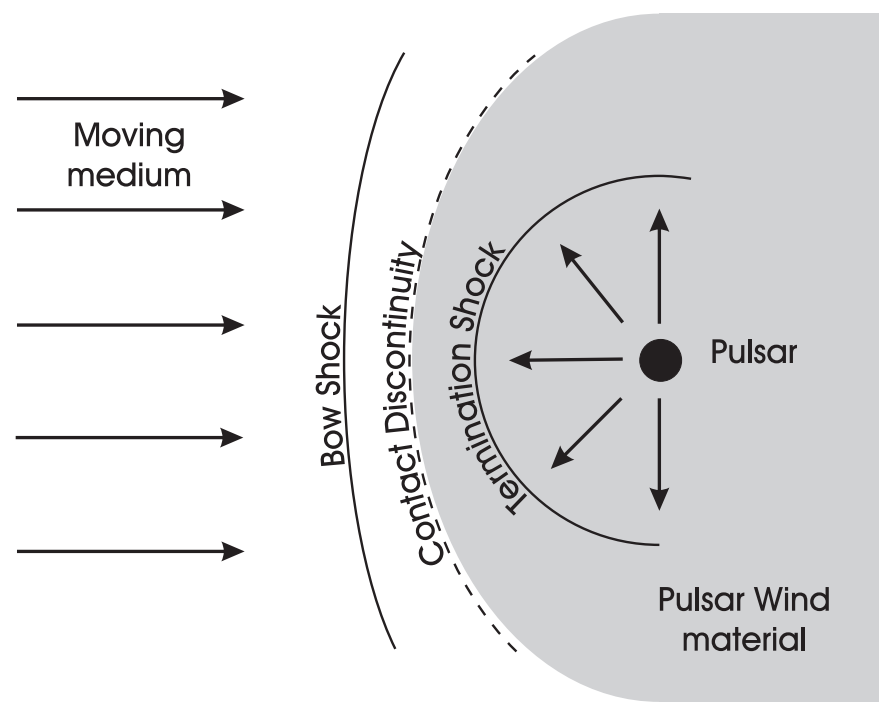

Fig. 2. Configuration of the pulsar wind nebula moving through a uniform medium, as seen in the rest frame of the pulsar.

\section{Numerical simulations of the PWN bow shock}

\subsection{Simulation method}

We simulate a pulsar wind using the Versatile Advection Code ${ }^{1}$ (VAC), a general-purpose software package developed initially by G. Tóth at the Astronomical Institute in Utrecht (Tóth 1996; Tóth \& Odstrčil 1996). The configuration is depicted in Fig. 2, showing both shocks which are of interest: the pulsar wind termination shock and the bow shock bounding the PWN. This system is assumed to be axially symmetric around the direction of motion of the pulsar. Out of the several choices for discretizing the equations of hydrodynamics in conservative form available in VAC, we use a shock-capturing, Total-VariationDiminishing Lax-Friedrich scheme (Tóth \& Odstrčil 1996). Our simulations have been performed in two dimensions with axial symmetry, using a cylindrical coordinate system $(R, z)$. We use continuous boundary conditions everywhere, except at the axis of symmetry where symmetric boundary conditions are used for density, total energy and the momentum component $p_{z}$ and anti-symmetric boundary conditions for $p_{R}$.

\subsection{Initialising a pulsar wind}

The current version of the hydrodynamics code uses one fluid with a single equation of state in the computational domain. For simplicity, and accuracy in the non-relativistic part of the flow, our calculations use a single fluid with one specific heat ratio, i.e. $\gamma=5 / 3$. Since the pulsar wind is most likely a mixture of electrons, positrons, magnetic fields and possibly some nuclei, our calculation can not treat the efficient cooling that could possibly result from the radiation losses (e.g. through synchrotron radiation) of the leptons in the mixture. Such cooling could lead to the collapse of the shocked wind material into a thin sheet.

The pulsar wind is modeled by continuously depositing thermal energy at a constant rate $L$ (the spindown

\footnotetext{
1 See http://www .phys.uu.nl/ ${ }^{\text {toth/ }}$
} 
Table 1. Simulation parameters.

\begin{tabular}{|l|c|}
\hline \hline & Simulation \\
\hline & \\
Pulsar wind luminosity $L_{0}(\mathrm{erg} / \mathrm{s})$ & $1.3 \times 10^{34}$ \\
Mass density $\rho_{0}\left(\mathrm{~g} / \mathrm{cm}^{3}\right)$ & $1.0 \times 10^{-24}$ \\
Terminal Velocity $v_{\infty}(\mathrm{cm} / \mathrm{sec})$ & $3.0 \times 10^{9}$ \\
Velocity Medium $V_{\text {rel }}(\mathrm{km} / \mathrm{sec})$ & 500.0 \\
Mach Number Pulsar $\mathcal{M}_{\mathrm{psr}}$ & 3.13 \\
Number of grid cells $($ in r-direction) & 300 \\
Number of grid cells $($ in z-direction) & 180 \\
Grid size $(\mathrm{pc})($ in r-direction) & 0.1 \\
Grid size $(\mathrm{pc})($ in z-direction) & 0.06 \\
\hline \hline
\end{tabular}

luminosity) in a small volume, together with an associated mass injection $\dot{M}_{\mathrm{pw}}$. The hydrodynamics code itself then develops a thermally driven wind with terminal velocity $v_{\infty}$ which is subsequently randomised at a termination shock. In order to increase the computationally efficiency, we take the mechanical luminosity $L$ and mass deposition rate $\dot{M}_{\mathrm{pw}}$ such that the terminal velocity of the wind as determined from these two parameters,

$v_{\infty}=\sqrt{2 L / \dot{M}_{\mathrm{pw}}}$

is much larger than the other velocities of interest. This choice will result in the correct global behaviour of the PWN. This method is similar to the method as described in van der Swaluw et al. (2001). We employ a non-uniform grid with highest resolution near the pulsar in order to fully resolve the pulsar wind region. The non-uniform grid is chosen in such a way that the difference in size between adjacent grid cells is always less than $5 \%$, and the refinement ratio between the largest and smallest cells equals 5 . The radius of the termination shock, given by Eq. (18) in the relativistic case, is replaced by its nonrelativistic equivalent,

$R_{\mathrm{ts}} \approx \eta\left(\frac{L}{2 \pi \rho_{\mathrm{ism}} V_{\mathrm{psr}}^{2} v_{\infty}}\right)^{1 / 2}$.

This approach yields roughly the correct value even for fully relativistic winds as long as the luminosity $L$ rather than the rest-mass flux $\dot{M}$ is employed to calculate the pressure behind the termination shock.

\subsection{Steady PWN bow shock in a uniform medium}

Our calculations are performed in the pulsar rest frame. The pulsar wind nebula is allowed to evolve in a uniform medium, moving at a constant speed $V_{\mathrm{psr}}$ at large distances from the pulsar. This medium represents the interior of the supernova remnant (shocked ISM) close to the blast wave. The velocity $V_{\mathrm{psr}}$ is supersonic with respect to the internal sound speed of the

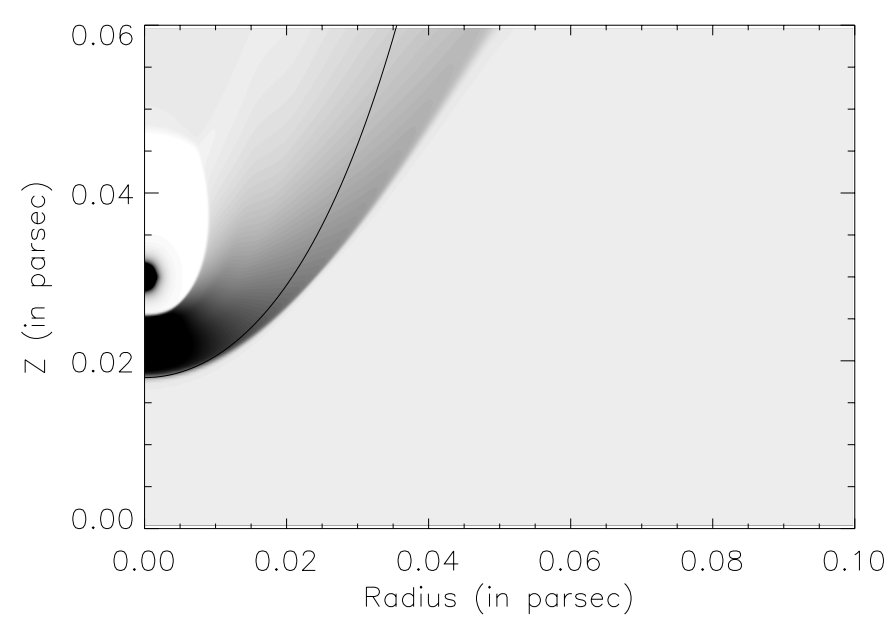

Fig. 3. Comparison between the numerical result for the bow shock with a low Mach Number $(\mathcal{M}=7 / \sqrt{5} \approx 3.13)$ with the profile given by Wilkin (1996) for $\mathcal{M} \gg 1$ (solid line). The grey scale represents the pressure.

medium so that a bow shock develops around the PWN. We let the hydrodynamics code evolve the system until the large-scale flow is steady.

This simulation has been performed with parameters as denoted in Table 1. In order to determine when the system is steady, we employ the prescription of Tóth et al. (1998). This prescription compares all $N_{\text {var }}$ flow variables at time $t_{n}$, denoted by $U_{\alpha i}^{n}$ at grid point $i$, with their values at the previous time $t_{n-1}$. We then calculate the residual Res defined as

$\operatorname{Res} \equiv \sqrt{\frac{1}{N_{\mathrm{var}}} \sum_{\alpha=1}^{N_{\mathrm{var}}} \frac{\sum_{i}\left[U_{\alpha i}^{n+1}-U_{\alpha i}^{n}\right]^{2}}{\sum_{i}\left[U_{\alpha i}^{n}\right]^{2}}}$,

and halt the calculation when Res has a value less than a predetermined critical value, typically Res $=10^{-4}$.

Wilkin (1996) has given an analytical equation for the geometry of a stellar wind bow shock. His solution, for the distance $r$ to the wind source in terms of the polar angle $\theta$ with respect to the symmetry axis, reads:

$\frac{r(\theta)}{R_{0}} \equiv \frac{1}{\sin \theta} \sqrt{3\left(1-\frac{\theta}{\tan \theta}\right)}$.

Here $R_{0} \approx R_{\mathrm{bs}}$ is the standoff distance of the bow shock on the symmetry axis $(\theta=0)$. We compare our morphology with Wilkin's result, where we equate $R_{0}$ to the standoff distance of the bow shock in the simulations. This is depicted in Fig. 3. The asymptotic cone of Wilkin's solution is seen to be significantly narrower than that of the bow shock in the simulation. This is because his solution balances only the ram pressures of the wind and the ambient medium, i.e. it corresponds to the limit $\mathcal{M}_{\mathrm{psr}} \gg 1$, while in our case the Mach number is moderate: $\mathcal{M}_{\mathrm{psr}} \approx 3.13$.

Figures 4 and 5 show density profiles of the PWN bow shock of a pulsar moving through a uniform medium. One can see the contact discontinuity, midway between the termination shock and the bow shock, separating the shocked pulsar wind material and the much denser shocked ISM. The synchrotron 


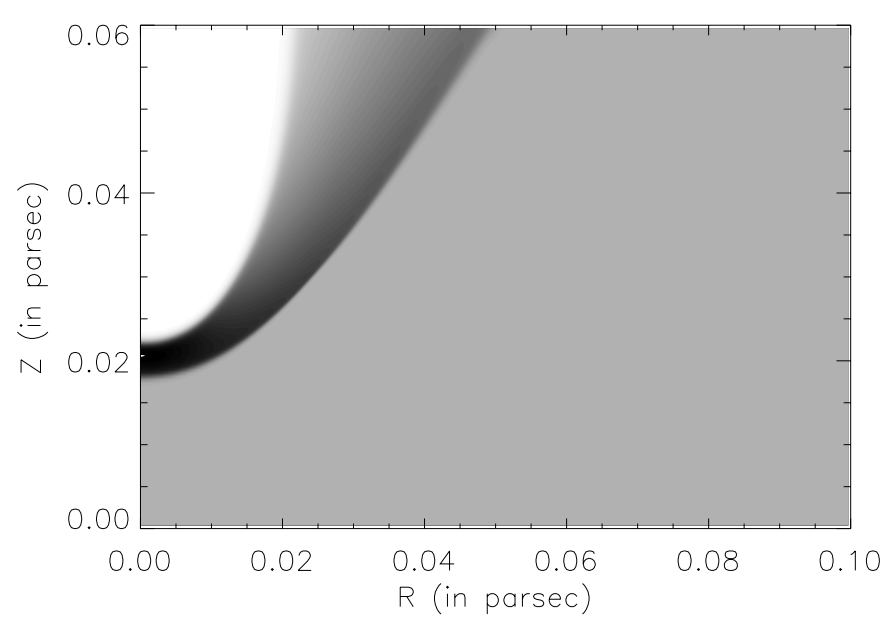

Fig. 4. Gray-scale representation of the density distribution of a PWN and bow shock with the parameters as denoted in Table 1 .

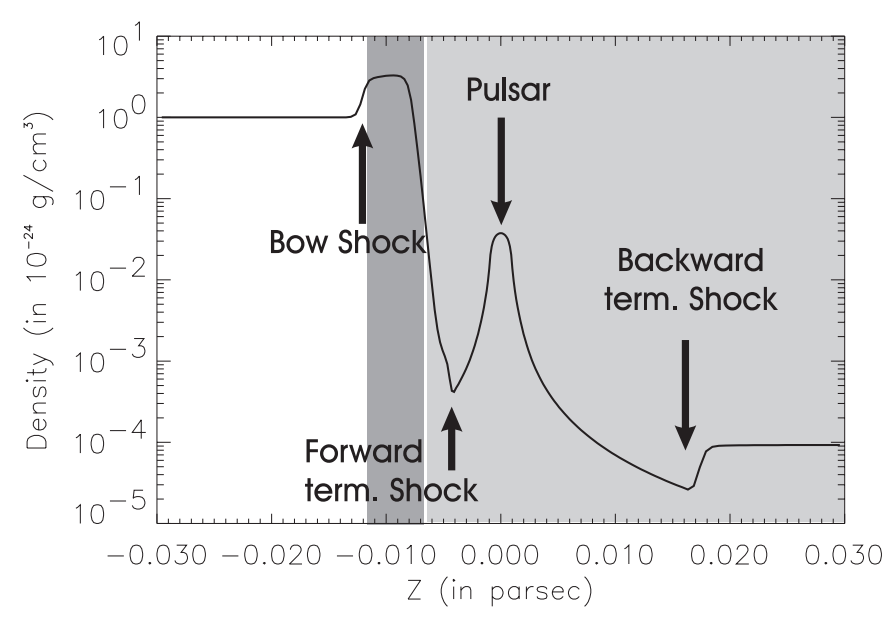

Fig. 5. Density profile of the PWN and bow shock along the $z$-axis of Fig. 4. The location of the Pulsar, forward and backward termination shocks and of the bow shock are indicated. The region containing both freely expanding and shocked pulsar wind material is shaded light gray, and the region containing shocked interstellar material is shaded a darker gray.

emission of the plerionic PWN is expected to come from the shocked pulsar wind material, whereas the material swept up by the bow shock can show up as $\mathrm{H} \alpha$ emission.

Figure 6 shows the pressure distribution and Fig. 7 shows the pressure profile along the symmetry axis. One can see the pulsar wind, originating at the pulsar position $z=0$, and its termination shock at $z \approx-0.005 \mathrm{pc}$ ahead of the pulsar in the direction of motion, and at $z \approx 0.017 \mathrm{pc}$ behind the pulsar. The bow shock bounding the PWN is located at $z \approx-0.012$ pc. The region between the pulsar wind termination shock and the bow shock is almost isobaric. As shown by van der Swaluw et al. (2001), this is also the case for a PWN around a stationary pulsar located at the center of the SNR.

The standoff distances of both the forward and backward termination shocks can be determined by specifying the confining pressure $P_{\text {ts }}$ in Eq. (15). One can use the pressure behind the tip of the bow shock $P_{\mathrm{bs}}$, as defined in Eq. (16), as the

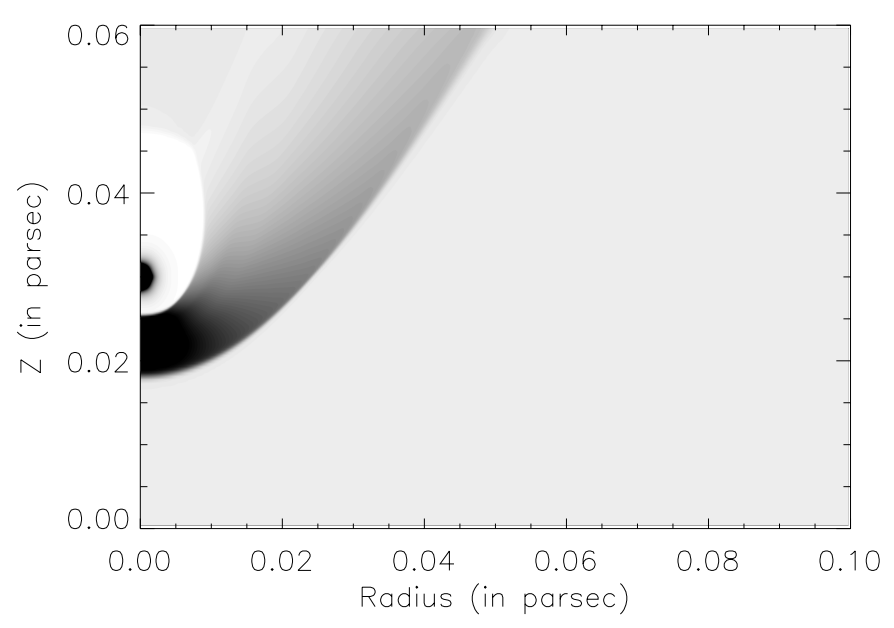

Fig. 6. Gray-scale representation of the pressure distribution of a PWN bow shock with the same parameters as in Fig. 4.

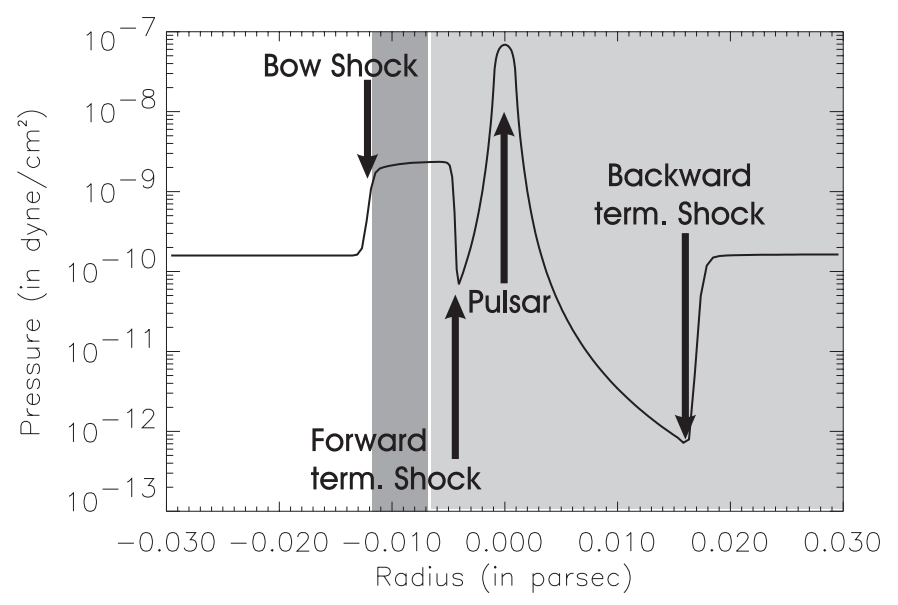

Fig. 7. Pressure profile of a PWN and bow shock along the $z$-axis of Fig. 6. Shading is as in Fig. 5.

typical pressure downstream of the forward termination shock, which yields the forward standoff distance:

$R_{\mathrm{ts}}^{\mathrm{fw}} \approx\left(\frac{L}{6 \pi P_{\mathrm{bs}} c}\right)^{1 / 2}$.

The backward termination shock sees a pressure roughly equal to the typical pressure of the (unperturbed) surrounding medium, since the bow shock becomes rather weak: $P_{\mathrm{ts}} \sim P_{\text {ext }}$. This yields the following standoff distance for the backward termination shock:

$R_{\mathrm{ts}}^{\mathrm{bw}} \approx\left(\frac{L}{6 \pi P_{\mathrm{ext}} c}\right)^{1 / 2}$,

and the ratio of the forward and backward termination shock distances is

$\frac{R_{\mathrm{ts}}^{\mathrm{fw}}}{R_{\mathrm{ts}}^{\mathrm{bw}}} \approx\left(\frac{P_{\mathrm{ext}}}{P_{\mathrm{bs}}}\right)^{1 / 2}$.

Using Eq. (16) with $\mathcal{M}_{\mathrm{psr}}=7 / \sqrt{5}$ (Eq. (10)) and $P_{\mathrm{sh}}=P_{\text {ext }}$ together with a specific heat ratio $\gamma=5 / 3$ as used in the simulations, the full Rankine-Hugoniot relations yield $P_{\mathrm{m}}=12 P_{\text {ext }}$, and we obtain a ratio $R_{\mathrm{ts}}^{\mathrm{fw}} / R_{\mathrm{ts}}^{\mathrm{bw}}=1 / 2 \sqrt{3} \approx 0.29$, a similar value as is observed in the Figs. 5 and 7. 


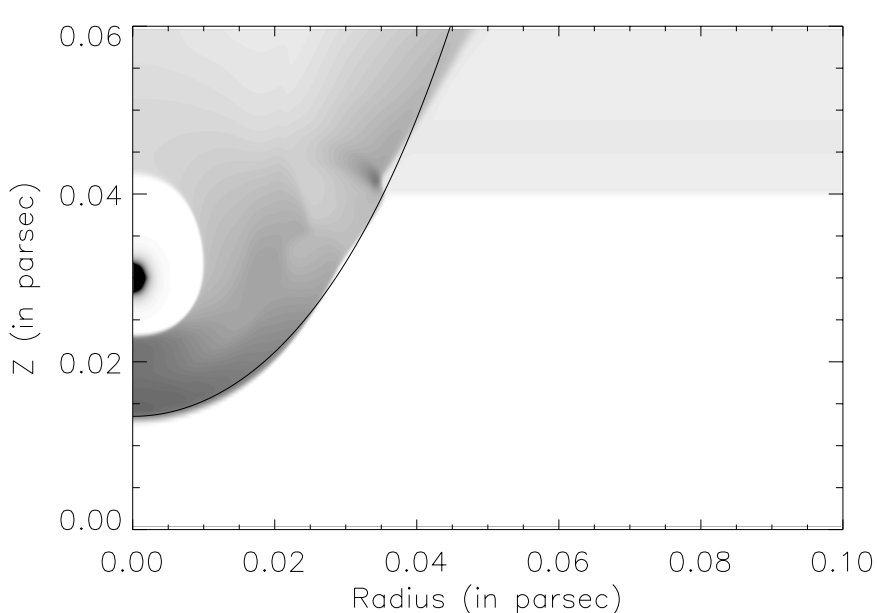

Fig. 8. Pressure distribution of a PWN bow shock with parameters as denoted in Table 1. Here the PWN is interacting with the shell of the SNR, modeled as a plane-parallel shock. The profile given by Wilkin (1996) for $\mathcal{M} \gg 1$ (solid line) agrees well with the bow shock profile in the ISM.

\subsection{Interaction of the PWN with a shock}

In this section we present results of the break-through of the PWN bow shock when it crosses the shell of its supernova remnant. The simulation is performed once more in the rest frame of the pulsar. We initialise a steady-state configuration of the PWN bow shock as described above, with the same parameters as denoted in Table 1. Next we use the Rankine-Hugoniot relations to initiate a strong shock front (with Mach number $\mathcal{M} \sim 100$ ) near the bottom (low $z$ ) end of the grid, with which the pulsar wind bow shock then catches up in accordance with Eqs. (7)-(10). As stated in Sect. 2, the PWN bow shock is much smaller than the radius of the SNR, so we can safely approximate the SNR blast wave as a plane-parallel strong shock.

At the end of the simulation, when the strong shock is almost at the upper boundary of the grid, numerical instabilities arise. Therefore we stop the simulation after the configuration as shown in the Figs. 8-10, when the influence of the numerical instabilities are not influencing the solution too strongly.

Figures 8 and 9 show the system, some time after the SNR shock has passed the head of the bow shock. Figure 8 shows the pressure distribution and Fig. 9 the density distribution. Figure 10, which compares the density profile along the $z$-axis before and after the passage of the supernova blast wave, shows that the pulsar wind nebula expands, roughly by a factor 1.5 , after it leaves the SNR. This reflects the reduction in the confining ram-pressure, as calculated in Sect. 2. Figure 8 also shows that the bow shock shape in the ISM now closely fits the analytical result of Wilkin (1996). This is because the pulsar wind bow shock has crossed the supernova blast wave, its Mach number with respect to the interstellar medium is much larger than unity. This agreement is all the more remarkable given that, strictly speaking, the assumptions under which Wilkin's solution is derived (geometrical thinness due to effective cooling of the post-bow shock material and complete mixing of the post-shock fluids) are not fulfilled in pulsar bow-shock nebulae (Bucciantini \& Bandiera 2001). The cooling time of the

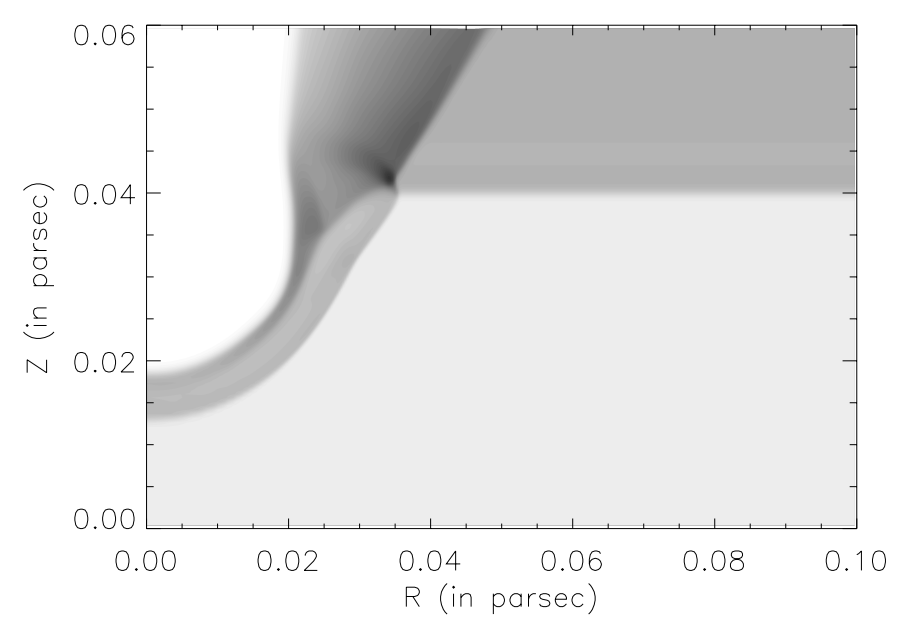

Fig. 9. Density distribution of the PWN bow shock-SNR interaction corresponding to the pressure distribution of Fig. 8.

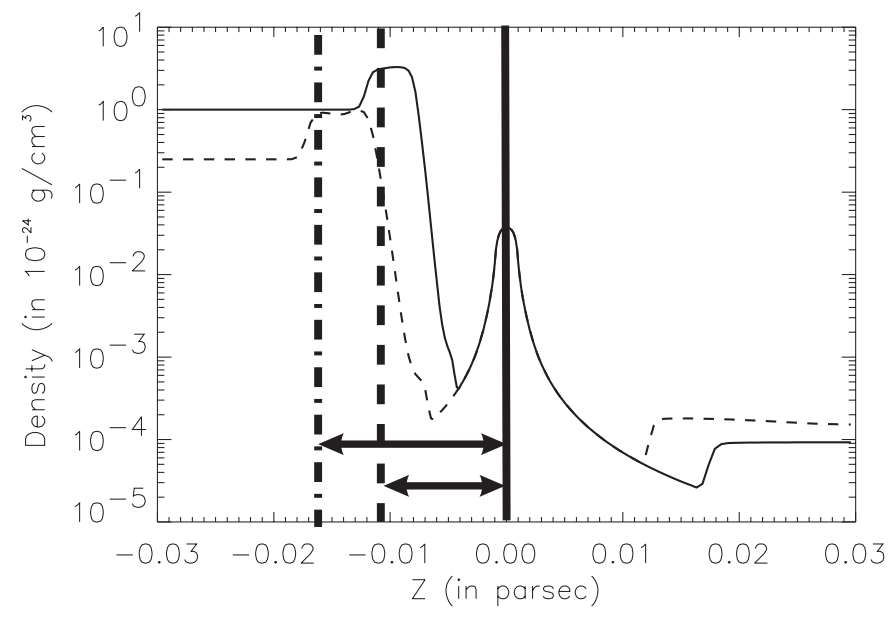

Fig. 10. Density profile of the PWN bow shock along the $z$-axis. In this figure the pulsar's position corresponds to $Z=0$. The solid curve denotes the density profile before interacting with the SNR shock. The dashed curve denotes the density profile well after the SNR shock has passed the head of the bow shock. The dashed line and the dashdot line give the position of the bow shock around the PWN before and after the passage of the supernova blast wave. The PWN expands roughly by a factor 1.5 .

shocked interstellar gas is much longer $\left(\sim 10^{7} \mathrm{yr}\right.$ for typical parameters) than the age of the system, so the "rapid cooling" assumption is not justified. However, the layer of shocked ISM remains relatively thin. This may explain why the shape of the bow shock still follows Wilkin's solution. Mixing can not be observed in these simulations as the assumption of axial symmetry suppresses some instabilities, and since the numerical scheme used is rather diffusive.

During the interaction between the pulsar wind and the shell of the SNR, the PWN bow shock and the SNR blast wave intersect. This intersection produces an additional pressure gradient which results in an accumulation of mass. The resulting density enhancement is of order $\sim 2.6$ with respect to the shocked ISM material downstream of the SNR blast wave. The pressure enhancement is of order $\sim 0.83$ with respect to the head of the bow shock. Both enhancements can be seen as dark 
spots at the region of intersection in the Figs. 8 and 9. When the bow shock moves through the shell of the remnant it encounters the unshocked ISM. The ambient density is reduced by a factor 4 , which results in a similar density reduction behind the bow shock.

\section{Conclusions}

We have considered the case of a pulsar wind breaking through the shell of a SNR in the Sedov-Taylor stage. Only highvelocity pulsars reach the edge of the SNR while the latter is still in the Sedov stage of its evolution. At the moment of break-through, the ratio of the pulsar velocity and SNR expansion speed is $V_{\mathrm{psr}} / V_{\mathrm{snr}}=5 / 2$, and the Mach number associated with the pulsar motion equals $\mathcal{M}_{\mathrm{psr}}=7 / \sqrt{5}$. These conclusions are independent of the explosion energy $E_{\mathrm{snr}}$ or the pulsar speed $V_{\mathrm{psr}}$.

Our simulations show that the break-through of the PWN does not lead to a significant disruption. The reduction of stagnation pressure by about $50 \%$ leads to a moderate expansion of the PWN where its radius increases by a factor $\sim 1.5$. The latter result was also obtained analytically in Eqs. (16)-(18).

There is good agreement between our numerical results and analytical estimates, based on pressure balance arguments, for the size of the bow shock surrounding the PWN. The only clear indication of the interaction between the PWN bow shock and the SNR (Sedov-Taylor) blast wave is a density and pressure enhancement at the intersection of these two shocks.

In a subsequent paper we will consider the effects of the energetic particles which were injected by the pulsar wind into the surroundings; some preliminary results of this investigation can be found in van der Swaluw et al. (2002).

Acknowledgements. The Versatile Advection Code was developed as part of the Massief Parallel Rekenen (Massive Parallel Computing) program funded by the Dutch Organisation for Scientific Research (NWO). The authors thank Dr. S. Falle (Dept. of Applied Mathematics, University of Leeds) for his assistance. We thank an anonymous referee for his helpful comments. EvdS was supported by the European Commission under the TMR programme for this project, contract number ERB-FMRX-CT98-0168. Y.A.G. acknowledges support from The Netherlands Organisation for Scientific Research (NWO) through GBE/MPR grand 614-21-008, and a Marie Curie Fellowship from the European Commission, contract number HPMFCT-2000-00671.

\section{References}

Arzoumanian, Z., Chernoff, D. F., \& Cordes, J. M. 2002, ApJ, 568, 289

Blondin, J. M., Wright, E. B., Borkowski, K. J., \& Reynolds, S. P. 1998, ApJ, 500, 342

Bucciantini, N., \& Bandiera, R. 2001, A\&A, 375, 1032

Cioffi, D. F., McKee, C. F., \& Bertschinger, E. 1988, ApJ, 334, 252

Cordes, J. M., Romani, R. W., \& Lundgren, S. C. 1993, Nature, 362, 133

Frail, D. A., \& Kulkarni, S. R. 1991, Nature, 352, 785

Frail, D. A., Goss, W. M., \& Whiteoak, J. B. Z. 1994, ApJ, 437, 781

Gaensler, B. M., \& Frail, D. A. 2000, Nature, 406, 158

Gallant, Y. A., van der Swaluw, E., Kirk, J. G., \& Achterberg, A. 2002, in Neutron Stars in Supernova Remnants, ASP Conf. Proc., ed. P. O. Slane, \& B. M. Gaensler, 99

Hansen, B. M. S., \& Phinney, E. S. 1997, MNRAS, 291, 569

Harrison, P. A., Lyne, A. G., \& Anderson, B. 1993, MNRAS, 261, 113

Kennel, C. F., \& Coroniti, F. V. 1984, ApJ, 283, 694

Lyne, A. G., \& Lorimer, D. R. 1994, Nature, 369, 127

Migliazzo, J. M., Gaensler, B. M., Backer, D. C., et al. 2002, ApJ, 567, L141

Romani, R. W., Cordes, J. M., \& Yadigaroglu, I. A. 1997, ApJ, 484, L137

Shull, J. M., Fesen, R. A., \& Saken, J. M. 1989, ApJ, 346, 860

Strom, R. G. 1987, ApJ, 319, L103

Tóth, G. 1996, Astrophys. Lett. \& Comm., 34, 245

Tóth, G., \& Odstrčil, D. 1996, J. Comp. Phys., 128, 82

Tóth, G., Keppens, R., \& Botchev, M. A. 1998, A\&A, 332, 1159

van der Swaluw, E., Achterberg, A., Gallant, Y. A., \& Tóth, G. 2001, A\&A, 380, 309

van der Swaluw, E., Achterberg, A., \& Gallant, Y. A. 2002, in Neutron Stars in Supernova Remnants, ASP Conf. Proc., ed. P. O. Slane, \& B. M. Gaensler, 135

Wilkin, F. P. 1996, ApJ, 459, L31 